\title{
Der Schuman-Plan und die Zukunft der Europäischen Union
}

\author{
Wilfried Loth*
}

60 Jahre nach der Regierungserklärung vom 9. Mai 1950, in der der französische Außenminister Robert Schuman vorschlug, ,die gesamte deutsche und französische Kohle- und Stahlproduktion einer gemeinsamen Hohen Behörde zu unterstellen, in einer Organisation, die für die Beteiligung der anderen europäischen Länder offen ist“", liegen die Anfänge der Europäischen Gemeinschaft für Kohle und Stahl (EGKS) im Nebel mythischer Vorzeit. Warum gerade Kohle und Stahl? Warum 1950? Warum gerade sechs Gründungsmitglieder, die den ersten Vertrag zu supranationaler Gemeinschaftsbildung in Europa am 18. April 1951 unterzeichneten? Selbst in den Gründerstaaten - Frankreich, die Bundesrepublik Deutschland, Belgien, die Niederlande, Luxemburg und Italien - werden nur Wenige diese Fragen einigermaßen verlässlich beantworten können. Erst recht dürfte die Gründungsgeschichte der heutigen Europäischen Union den vielen ,newcomern' fernliegen, die der Gemeinschaft zu unterschiedlichen Zeiten beigetreten sind. Die Europäische Kommission hat gerade beschlossen, die Förderung des Steinkohle-Bergbaus im Jahr 2014 ganz auslaufen zu lassen; für Schuman ist ein Seligsprechungsprozess der katholischen Kirche im Gang.

Wenn die Politiker und Bürger der Europäischen Union ein Selbstbewusstsein als Unionsbürger entwickeln sollen, ist es aber wichtig, realistische Vorstellungen von den Anfängen dieser Union zu haben. Die Geschichtswissenschaft hat viel dafür getan, solche Vorstellungen zu entwickeln. Weil Zeithistoriker immer auch Zeitgenossen sind, kam es dabei zu mancherlei Kontroversen: Der Mythos vom europäischen Idealismus der Gründerväter wurde mit Gegenmythen vom ewigen Ringen nationaler Interessen bekämpft. Unterdessen liegen aber genügend Argumente und Fakten auf dem Tisch, ${ }^{2}$ um eine abgewogene Bilanz wagen zu können, die die Gründung der EGKS aus dem Nebel der Mythenbildung holt. Sie soll hier in aller Kürze versucht werden.

\section{Der Plan und seine Vorläufer}

Um die Bedeutung des Schuman-Plans zu verstehen, muss man zunächst zwischen allgemeinen Traditionslinien und Vorläufern des spezifischen Projekts unterscheiden. Der Schuman-Plan lässt sich in zweierlei Traditionen einordnen: die Tradition der Bemühungen um eine Wiedervereinigung des schwerindustriellen Komplexes von der Ruhr bis nach Lothringen, der in der Zeit des deutschen Kaiserreichs entstanden war, und die Tradition der Bemü-

* Prof. Dr. Wilfried Loth, Lehrstuhl für Neuere und Neueste Geschichte an der Universität Duisburg-Essen, Präsident der Verbindungsgruppe der Historiker bei der Kommission der Europäischen Gemeinschaften.

1 Zitiert nach: Horst Möller/Klaus Hildebrand (Hrsg.): Die Bundesrepublik Deutschland und Frankreich: Dokumente 1949-1963. Band 1: Außenpolitik und Diplomatie, München 1997, S. 225-227.

2 Vgl. Klaus Schwabe (Hrsg.): Die Anfänge des Schuman-Plans 1950/51, Baden-Baden 1988; Ulrich Lappenküper: Der Schuman-Plan. Mühsamer Durchbruch zur deutsch-französischen Verständigung, in: Vierteljahrshefte für Zeitgeschichte 3/1994, S. 403-445; Gilbert Trausch: Der Schuman-Plan zwischen Mythos und Realität. Der Stellenwert des Schuman-Planes, in: Rainer Hudemann/Hartmut Kaelble/Klaus Schwabe (Hrsg.): Europa im Blick der Historiker, Historische Zeitschrift. Beihefte, München 1995, S. 105-128; Armin Heinen: Frankreich, Großbritannien und der Schumanplan - oder - Die „Entzauberung der Welt“ durch die Zeitgeschichte, in: Guido Müller (Hrsg.): Deutschland und der Westen. Festschrift für Klaus Schwabe zum 65. Geburtstag, Stuttgart 1998, S. 65-75; Andreas Wilkens (Hrsg.): Le Plan Schuman dans l'Histoire. Intérêts nationaux et projet européen, Brüssel 2004. 
hungen um eine europäische Einigung, die in Reaktion auf die Katastrophen des Zweiten Weltkriegs wieder aufgenommen und verstärkt worden waren. Sowohl die wechselseitige Abhängigkeit der beiden schwerindustriellen Zentren als auch die wachsende Dysfunktionalität eines Systems absolut souveräner Nationalstaaten in Europa haben vielfältige Einigungsinitiativen hervorgerufen, auf wirtschaftlichem Gebiet wie auf politischem.

In beiden Bereichen wurde die Frage nach der Form der Einigung durchaus unterschiedlich beantwortet, ebenso die Frage nach den Bedingungen der Einigung und die Frage nach der Zusammensetzung der europäischen Gemeinschaft. Die Fülle der Vorschläge und ehrgeizigen Projekte reicht von einer Verständigung über Exportbeschränkungen bis zur vollständigen Beseitigung von Wirtschaftsgrenzen, von Absprachen zwischen Großunternehmen bis zu völkerrechtlichen Verträgen, von der hegemonialen Vereinigung unter der Federführung einer Vormacht bis zur Einigung zwischen gleichberechtigten Partnern, von der Gestaltung durch ein zentrales Entscheidungszentrum bis zur ständigen Konzertierung zwischen Gründern, die die Herren der Verträge bleiben. Die Geschichte der europäischen Idee und der europäischen Einigung sind durch die Debatte und den Konkurrenzkampf zwischen diesen unterschiedlichen Formen der Gestaltung, der Einigung und der Integration gekennzeichnet.

Im Schuman-Plan überschneiden sich die Traditionslinien der Wiedervereinigung der Schwerindustrie beiderseits des Rheins und der politischen Konstruktion Europas. Was ihn von den vielen anderen Einigungsprojekten unterscheidet, ist die Idee einer gemeinsamen supranationalen Lenkung der Schwerindustrien Ostfrankreichs und Westdeutschlands. Damit schlägt er die Vereinigung der schwerindustriellen Zentren in einem spezifischen geopolitischen Kontext vor: dem Aufstieg Westdeutschlands zum unverzichtbaren Partner des Westens im entstehenden Kalten Krieg. Gleichzeitig propagiert er die Einigung durch Einrichtung einer politischen Autorität auf einer Ebene oberhalb der Nationalstaaten, die damit deren Souveränität beschränkt. In dieser Hinsicht folgt der Schuman-Plan einer Linie unmittelbarer Vorläufer, die mit einem Vorschlag von André Philip beginnt, den er am 30. November 1948 im Namen der sozialistischen Fraktion in der französischen Nationalversammlung präsentiert hat und der auf die Schaffung eines ,intereuropäischen öffentlichen Dienstes für Kohle und Stahl“ zielte, zuständig für die Bereiche der Produktion, der Investition und der Festsetzung der Preise. ${ }^{3}$ Ihm folgte ein ähnlicher Vorschlag von Karl Arnold, dem Ministerpräsidenten des Landes Nordrhein-Westfalen, aus Anlass seiner Neujahrsansprache 1949. ${ }^{4}$ Im April 1949 wurde er von der Wirtschaftskonferenz der Europäischen Bewegung übernommen, im Hinblick auf den Stahlbereich im Dezember 1949 auch von einem Unterausschuss des Europarats. Insbesondere die zusehends dringlicher werdende Kampagne von Philip bereitete die politischen Milieus des westlichen Europas auf die Initiative vom 9. Mai 1950 vor. ${ }^{5}$

3 Journal Officiel de la République française. Assemblée nationale. Débats parlementaires, 01.12.1948, S. 73127316. Siehe Wilfried Loth: Sozialismus und Internationalismus. Die französischen Sozialisten und die Nachkriegsordnung Europas 1940-1950, Stuttgart 1977, S. 218ff.; zum Kontext siehe Wilfried Loth: André Philip und das Projekt eines sozialistischen Europas, in: Martin Greschat/Wilfried Loth (Hrsg.): Die Christen und die Entstehung der Europäischen Gemeinschaft, Stuttgart 1994, S. 189-202.

4 Walter Lipgens/Wilfried Loth (Hrsg.): Documents on the History of European Integration. Band 3: The Struggle for European Union by Political Parties and Pressure Groups in Western European countries 19451950, Berlin/New York 1988, S. 519-521; Jürgen Mittag/Claudia Hülsken: Die Vernetzung des Ruhrgebiets in Europa: Der „Arnold-Plan“ als Wegmarke europäischer Integration, in: Forum Industriedenkmalpflege und Geschichtskultur 1/2007, S. 13-20.

5 Loth: Sozialismus, 1977, S. 228, 241, 263. 


\section{Eine französische Initiative}

Angesichts dieser Vorgeschichte kommt Jean Monnet nicht so sehr die Rolle eines ,Inspirators ' des Schuman-Plans zu, wie sie in der herkömmlichen Schuman-Plan-Mythologie beschrieben wird. Stattdessen sollte man ihn als „Geburtshelfer“" charakterisieren, wie René Girault zu Recht vorgeschlagen hat. ${ }^{6}$ Monnets Verdienst ist es, Schuman zu einem bestimmten Zeitpunkt von der Notwendigkeit überzeugt zu haben, das Projekt zu lancieren: im letzten Moment vor einer Konferenz der Außenminister der drei westlichen Besatzungsmächte am 11. und 12. Mai 1950, die eine Erhöhung der zulässigen westdeutschen Stahlproduktion beschließen sollte. Darüber hinaus hat er als Verhandlungsführer der französischen Delegation für eine entschlossene und effektive Umsetzung des französischen Vorschlags gesorgt. ${ }^{7}$ Er verfügte über das Gespür für den richtigen Augenblick, die Kunst, das Vertrauen der entscheidenden Politiker zu gewinnen, und den nötigen Scharfblick bei der Verfolgung des Wesentlichen. Diese Talente halfen ihm, den entscheidenden Schritt zu organisieren, der noch getan werden musste - auch wenn er in gewisser Weise schon in der Luft lag und der objektiven Lage Frankreichs im Kalten Krieg entsprach. Schumans Rolle bestand darin, dafür die politische Verantwortung zu übernehmen, das heißt die Risiken, die mit einem solchen Vorschlag verbunden waren, auf sich zu nehmen und sein politisches Schicksal mit dem Erfolg des Projektes zu verbinden. ${ }^{8}$ Insofern trägt der Schuman-Plan seinen Namen zu Recht.

Die Motive für diese Initiative der französischen Regierung sind gut bekannt, spätestens seit der Veröffentlichung eines grundlegenden Memorandums, das Monnet am 3. Mai 1950 Schuman vorgelegt hat. ${ }^{9}$ In erster Linie ging es darum, endlich das deutsche Problem zu lösen, wie es Schuman in einer persönlichen Aufzeichnung skizziert hat: „Deutsche Ressourcen und Energien - sich darauf beschränken, sie einzudämmen und zu bremsen, durch Verbote, Isolierung und feindliche Koalitionen, oder sie zur Geltung bringen, sie gemeinsam Zinsen abwerfen lassen zum Nutzen des ganzen vereinten Europa. "10 Die gemeinsame Erschließung entsprach nicht nur den Notwendigkeiten des europäischen Wiederaufbaus. Sie war umso dringender geboten, als die Entwicklung des Kalten Krieges die Orientierung an der Alternative der Eindämmung nicht länger zuließ: Die britischen und amerikanischen Verbündeten verlangten die Aufhebung der Beschränkung der westdeutschen Stahlproduktion auf 11,1 Millionen Tonnen pro Jahr und ihre Stabschefs drängten sogar schon auf die Aufstellung deutscher Truppen. Dass sich die Deutschen ihren Beitrag zur Verteidigung des Westens mit einer Aufhebung der einseitigen Kontrollen bezahlen lassen würden, war nur zu deutlich vorauszusehen.

Hinzu kam eine Überproduktionskrise in der Stahlindustrie, die nicht nur alle französischen Pläne zunichte machte, im Zuge des Wiederaufbaus zur ersten schwerindustriellen Macht des europäischen Kontinents zu avancieren, sondern auch die Gefahr einer raschen Rückkehr zur industriellen Hegemonie Deutschlands mit sich brachte - dank seiner Res-

6 René Girault: Interrogations, réflexions d'un historien sur Jean Monnet, l'Europe et les chemins de la Paix, in: Gérard Bossuat/Andreas Wilkens (Hrsg.): Jean Monnet, l'Europe et les chemins de la Paix, Paris 1999, S. 1319, hier S. 15.

7 Vgl. insbesondere die Berichte bei Lappenküper: Schuman-Plan, 1994; Éric Roussel: Jean Monnet. 18881979, Paris 1995, S. 518-566.

8 Zu Schuman vgl. die bahnbrechende Arbeit von Raymond Poidevin: Robert Schuman, homme d'État 18861963, Paris 1986, hier S. 244-263.

9 Le Monde, 09.05.1970, deutsche Übersetzung in: Gilbert Ziebura: Die deutsch-französischen Beziehungen seit 1945. Mythen und Realitäten, Pfullingen 1970, S. 195-200.

10 Undatierte Notiz im Nachlass Schuman, ermittelt von Trausch: Schuman-Plan, 1995, S. 113-114. 
sourcen an Kokskohle und der traditionellen Bevorzugung deutscher Stahlwerke bei der Belieferung mit dieser Kohle. In dieser Hinsicht könnte man den Schuman-Plan auch als ,zweiten Monnet-Plan ' bezeichnen - darauf bedacht, die Anstrengungen zur Modernisierung der französischen Industrie unter den neuen Bedingungen des Kalten Krieges fortzusetzen. ${ }^{11}$ Entsprechend standen ihm die französischen Stahlindustriellen, die sich für diese Modernisierung schlecht gerüstet fühlten, mehrheitlich skeptisch bis ablehnend gegenüber. ${ }^{12}$

Im Prinzip beinhaltete dieser zweite Monnet-Plan einen Verzicht auf die Ambitionen auf eine französische Führungsrolle auf dem europäischen Kontinent. Es scheint, dass sich Monnet und Schuman zum Zeitpunkt der Lancierung ihres Plans darüber nicht ganz im Klaren waren, nicht zuletzt weil die französischen Fortschritte bei der Kokserzeugung zur Fortdauer der Illusionen beitrugen. In einem Schreiben vom 9. September 1950 an Schuman, in dem Monnet das Problem der deutschen Wiederbewaffnung diskutiert, spricht er wiederholt von der „Führungsposition auf dem Kontinent, die Frankreich in der supranationalen Gemeinschaft einnehmen soll, die wir schaffen wollen." 13 Für die Parlamentarische Versammlung der EGKS sieht er ganz selbstverständlich eine überproportionale Gewichtung der französischen Stimmen vor. Dagegen präsentiert er die Preisgabe der entgegengesetzten deutschen Forderung nach einer Gewichtung gemäß dem jeweiligen Umfang der Kohle- und Stahlproduktion der beteiligten Länder und die Verständigung mit Konrad Adenauer über eine gleich starke Vertretung Frankreichs und der Bundesrepublik Deutschland als einen französischen Erfolg und als Ausweis des Gemeinschaftsgeistes. ${ }^{14}$ Tatsächlich spiegelt die Entwicklung des Schuman-Plans im Zuge der Verhandlungen das wachsende Gewicht der Bundesrepublik nach dem Beginn des Korea-Krieges wider. Monnet ist das aber nur teilweise bewusst. Auf jeden Fall spricht er darüber nicht.

Man kann den ursprünglichen Vorschlag der französischen Regierung daher als ein Übergangsprojekt betrachten, dessen technokratischer Charakter - die Schaffung einer Hohen Behörde ohne direkte Verbindungen mit den Nationalstaaten und ohne strikte Kontrollen eine Wahrheit verbirgt, die für Frankreich eher bitter ist. Wie Andreas Wilkens in einem Essay über das Verhältnis von Monnet und Adenauer geschrieben hat, ,bestand die Kraft des Schuman-Plans im Wesentlichen im Prinzip einer Gleichbehandlung der Bundesrepublik. "15 Es dürfte verständlich sein, dass die Anerkennung dieses Prinzips den französischen Initiatoren nicht leicht gefallen ist.

\section{Die deutsche Reaktion und die Rolle der USA}

Auf der anderen Seite war auch die Zustimmung der Bundesrepublik Deutschland zu dieser wechselseitigen Kontrolle keineswegs selbstverständlich. Gewiss bot der Schuman-Plan der Bundesrepublik die Perspektive einer Beendigung der einseitigen Kontrolle, das heißt einer Aufhebung der Internationalen Ruhrbehörde, der Beschränkungen der deutschen Stahl-

11 Die Wirtschaftsabteilung des französischen Außenministeriums hat das Projekt im Mai so bezeichnet. Siehe Trausch: Schuman-Plan, 1995, S. 114-115; als wissenschaftliche Analyse Constantin Goschler/Christoph Buchheim/Werner Bührer: Der Schumanplan als Instrument französischer Stahlpolitik. Zur historischen Wirkung eines falschen Kalküls, in: Vierteljahrshefte für Zeitgeschichte 2/1989, S. 171-206.

12 Siehe Sylvie Lefèvre-Dalbin: Les sidérurgistes français face au Plan Schuman: la réaction ambivalente de la maison De Wendel, 1950-1952, in: Wilkens: Plan Schuman, 2004, S. 197-217.

13 Fondation Jean Monnet pour l'Europe, Centre de recherches européennes (Hrsg.): Jean Monnet - Robert Schuman. Correspondance 1947-1953, Lausanne 1986, S. 53-55.

14 Jean Monnet: Mémoires, Paris 1976, S. 413-415; vgl. Andreas Wilkens: Jean Monnet, Konrad Adenauer et la politique européenne de l'Allemagne fédérale - Convergence et discordances (1950-1957), in: Bossuat/Wilkens: Jean Monnet, 2004, S. 147-201, hier S. 158.

15 Ebenda. 
produktion und des Besatzungsstatuts. Aber nichts verpflichtete die Deutschen, dafür eine gewiss wechselseitige, aber auch dauerhafte Kontrolle zu akzeptieren. Zudem war das Gleichgewicht wechselseitigen Vertrauens nur schwer aufrecht zu erhalten, das für das Gelingen einer solchen konstruktiven Lösung erforderlich war: Vertrauen in die demokratischen Tugenden des künftigen deutschen Partners auf der einen Seite, Vertrauen in das Fair Play der bisherigen Besatzungsmacht auf der anderen. Dieses Gleichgewicht konnte jederzeit gebrochen werden. Im Frühjahr 1950, insbesondere nach der Unterzeichnung der Konventionen zwischen Frankreich und dem Saarland vom 3. März, die die Währungs- und Zollunion zwischen beiden Ländern festschrieben, ${ }^{16}$ sah Adenauer diesen Moment immer näher kommen. Dies erklärt seine immer phantastischer werdenden Einigungsvorschläge und seine offenkundige Erleichterung, als Schuman ihn am 8. Mai 1950 noch vor den Mitgliedern der französischen Regierung über seine bevorstehende Initiative informierte.

Für den Erfolg dieser Initiative war der Beitrag von Adenauer also in hohem Maße entscheidend. Die Europa-Visionen des deutschen Bundeskanzlers zeichneten sich nicht gerade durch hohe Präzision aus. Aber gerade diese Flexibilität ließ seine Europapolitik so außerordentlich effektiv werden. Flexibilität galt für Adenauer nämlich nur auf der taktischen Ebene. Sie war an tiefgreifende Überzeugungen gebunden, die ihn von der Mehrzahl seiner deutschen Mitbürger unterschied. Adenauer wusste in hohem Maße um die Notwendigkeit, die Sicherheitsbedürfnisse der europäischen Nachbarn zu berücksichtigen, insbesondere die Sicherheitsbedürfnisse Frankreichs. Er war von der Notwendigkeit einer Emanzipation der Deutschen von der preußischen Vorherrschaft überzeugt, einer Westorientierung der Deutschen, einer Überwindung des Nationalismus in Deutschland und in Europa, einer westlichen Integration der Deutschen und einer , organischen Verflechtung' aller Europäer. Gleichzeitig war er von der Furcht vor der Sowjetunion dermaßen besessen, dass er in der Verfolgung seiner europäischen Politik keinen Moment im Hinblick auf die Einheit oder Wiedervereinigung Deutschlands zögerte.

Durch diese außerordentliche Verbindung von tiefgreifenden Überzeugungen und pragmatischer Flexibilität war Adenauer der ideale deutsche Gesprächspartner für die Westmächte im Hinblick auf die Schaffung des westlichen Bündnisses. ${ }^{17}$ Wie Klaus Schwabe gezeigt hat, hat Adenauer diese Rolle auch bei der Verwirklichung des Schuman-Plans gespielt, der sich durch die Macht der Umstände zum Grundstein der europäischen Konstruktion entwickelte. Die Furcht vor einer Verständigung zwischen Frankreich und der Sowjetunion sowie eines Rückfalls der USA in den Isolationismus ließen ihn jeden Vorschlag einer europäischen Kontrolle akzeptieren, wenn er der Bundesrepublik nur die Aussicht auf eine Gleichbehandlung in der Zukunft ließ. ${ }^{18}$ Adenauer wurde damit zu einem der ,Väter Europas‘. Wenn man diesem Ehrentitel einen präzisen Sinn geben will, ist es angebracht, ihn für die drei Männer zu reservieren, die es verstanden hatten, den Eckstein zu lancieren und zu errichten, also für Monnet, Schuman und Adenauer.

Diese Feststellungen führen zugleich zur Debatte zwischen Volker Berghahn, Werner Bührer und anderen über die Rolle der US-Regierung bei der Verwirklichung des SchumanPlans. ${ }^{19}$ Zweifellos haben die Amerikaner nur bei einem einzigen Punkt in die Erarbeitung

16 Vgl. Armin Heinen: Saarjahre. Politik und Wirtschaft im Saarland 1945-1955, Stuttgart 1996, S. 348-360.

17 Vgl. Wilfried Loth: Konrad Adenauer und die europäische Einigung, in: Mareike König/Matthias Schulz (Hrsg.): Die Bundesrepublik Deutschland und die europäische Einigung 1949-2000, Stuttgart 2004, S. 39-59.

18 Klaus Schwabe: L'Allemagne, Adenauer et l'option de l'intégration à l'Ouest, in: Wilkens: Plan Schuman, 2004, S. 81-105.

19 Vgl. Werner Bührer: Auf eigenem Weg. Reaktionen deutscher Unternehmer auf den Amerikanisierungsdruck, in: Heinz Bude/Bernd Greiner (Hrsg.): Westbindungen. Amerika in der Bundesrepublik, Hamburg 1999, S. 181-201; Volker Berghahn: Reconstruction et réorganisation. Le modèle américain, l'économie européenne de l'après-guerre et le Plan Schuman, 1941-1951, in: Wilkens: Plan Schuman, 2004, S. 107-121. 
des Vertrags über die Gemeinschaft für Kohle und Stahl eingegriffen, nämlich im Hinblick auf die Garantien für die Entflechtung der deutschen Industrie. In der Perspektive der amerikanischen Entscheidungsträger war dies aber der wesentliche Punkt im Projekt einer Restrukturierung der europäischen Industrie, die über eine Restrukturierung der deutschen Industrie erfolgen sollte. Bis hierhin erscheint die Argumentation von Berghahn überzeugend.

Die Feststellung eines amerikanischen Erfolgs rechtfertigt es freilich noch nicht, von einer Amerikanisierung der europäischen Industrie zu sprechen. Vielmehr kann man eine Übereinstimmung zwischen unterschiedlichen Modernisierungsanstrengungen registrieren, die zu einem gemeinsamen Erfolg führten. So wahrscheinlich es ist, dass Monnet ohne die amerikanische Unterstützung, ohne die energischen Interventionen von Hochkommissar John McCloy und dem sonstigen Druck, der von amerikanischer Seite ausgeübt wurde, die Garantien zur Entflechtung der deutschen Industrie niemals erhalten hätte, so richtig ist auch, dass McCloy ohne die Zustimmung Adenauers nicht in der Lage gewesen wäre, der deutschen Industrie seinen Standpunkt aufzuzwingen. Adenauers Furcht vor einer Rückkehr zum Isolationismus hat den Sieg der Koalition der Modernisierer ermöglicht - einer Koalition, die durch die beständigen Angriffe der Kommunisten nur noch verstärkt wurde. ${ }^{20}$

\section{Die Rolle der europäischen Partner}

Die Geschichte des Schuman-Plans ist also in erster Linie eine deutsch-französische Geschichte, die Geschichte eines de-facto-Friedensvertrags zwischen Frankreich und Deutschland. Dieser Friede konnte aber nicht ohne den Beitrag der anderen Partner der westeuropäischen Konstruktion geschlossen werden. Er lag aber auch in deren eigenem Interesse. Damit ist jede Geschichte der Anfänge der europäischen Konstruktion unvollständig, die sich auf die beiden Hauptakteure beschränkt.

Die Frage, die sich den europäischen Partnern der deutsch-französischen Konstruktion stellte, betraf die Vereinbarkeit des Interesses an einer Integration Westdeutschlands mit ihren sonstigen Interessen und Erfordernissen. Für Italien und die Beneluxstaaten war die Antwort auf diese Frage im Wesentlichen positiv - auch wenn sich Belgien eine beträchtliche Fortdauer des Rechts zu sichern wusste, seine überalterte Kohleindustrie zu subventionieren, und Italien darum bemüht war, die Freizügigkeit seiner Arbeitskräfte sowie die Festlegung einer Übergangsperiode bis zur vollen Marktintegration zu erhalten. Den Beneluxstaaten ist insbesondere die Klärung beziehungsweise Stärkung der Rolle der nationalen Regierungen in der definitiven Konstruktion der EGKS zu verdanken. Man kann damit mit Monnet und entsprechend den Ambitionen des niederländischen Außenministers Dirk Stikker eine Schwächung des supranationalen Prinzips sehen. ${ }^{21}$ Gleichwohl kommt den Vertretern der Niederlande, Luxemburgs und Belgiens das Verdienst zu, das Problem der demokratischen Kontrolle und Legitimation einer supranationalen Konstruktion mit aller Deutlichkeit zur Sprache gebracht zu haben. Was den Einfluss Italiens betrifft, so muss man mit Daniela Preda festhalten, dass der italienische Ministerpräsident Alcide de Gasperi wesentlich deutlicher als die anderen Verantwortlichen der Gründungsperiode sah, dass sich die EGKS auf Dauer nur als Beginn eines politischen Europas halten ließ.

20 Vgl. Ulrich Pfeil: La République démocratique allemande et le Plan Schuman, 1950-1952, in: Wilkens: Plan Schuman, 2004, S. 323-359.

21 Vgl. Marie-Thérèse Bitsch: La triple option de Paris: pour une Europe supranationale et sectorielle autour d'un noyau franco-allemand, in: Wilkens: Plan Schuman, 2004, S. 149-168; Pascaline Winand: Max Kohnstamm et le Plan Schuman: „On change les assiettes“ ou „Plus est en vous“, in: Wilkens: Plan Schuman, 2004, S. 361372. 
Die britische Antwort fiel, wie man weiß, negativ aus. Über diese Entscheidung gegen eine Beteiligung an den Anfängen der europäischen Konstruktion ist viel diskutiert worden und es mangelt nicht an Stimmen, die zumindest im Rückblick meinen, dass diese Entscheidung falsch gewesen sei. ${ }^{22}$ Die Beratungen in der britischen Regierung zeigen aber, dass die negative Antwort nicht von vorneherein feststand und dass die Akteure sie gleichwohl als eine konstruktive Antwort verstanden wissen wollten. Außenminister Ernest Bevin und die anderen Verantwortlichen an der Themse wussten, dass Großbritannien auf die deutschfranzösische Verständigung angewiesen war. Nachdem sie mit dem Versuch gescheitert waren, den Schuman-Plan ohne seine supranationale Dimension zu verwirklichen (was ihm den Kern seiner Nützlichkeit genommen hätte), vermieden sie es sorgsam, das Projekt der Sechs in irgendeiner Weise zu boykottieren. Eine ausgewogene Geschichte des SchumanPlans sollte auch diesen britischen Beitrag zum schließlichen Erfolg unterstreichen. ${ }^{23}$

Man muss hinzufügen, dass ein Europa ohne Großbritannien natürlich viel weniger attraktiv war und in der öffentlichen Meinung viel weniger Unterstützung hatte als ein Europa unter britischer Führung, wie es der Mehrheit der Europäischen Bewegung an der Schwelle zu den 1950er Jahren vorschwebte. Wie Anne Dulphy und Christine Manigand gezeigt haben, sperrte sich etwa die öffentliche Meinung in Frankreich gegen die Idee einer Partnerschaft mit Deutschland und zog ihr eine Partnerschaft mit Großbritannien bei Weitem vor. ${ }^{24}$ Der Schuman-Plan hat also keineswegs allgemeine Begeisterung hervorgerufen - was die politische Leistung Schumans und auch Adenauers umso bemerkenswerter erscheinen lässt. Ganz verständlich wird sie erst vor dem Hintergrund eines Netzwerks pro-europäischer Christdemokraten in Frankreich und Deutschland, die sich in ihren innerparteilichen Auseinandersetzungen wechselseitig unterstützten. ${ }^{25}$ In der geringen Akzeptanz eines Europas ohne Großbritannien dürfte aber auch der wesentliche Grund dafür liegen, warum von der EGKS kein direkter Weg zur politischen Föderation Europas führte, wie ihn de Gasperi für notwendig hielt, und warum das europäische Projekt nach dem verheißungsvollen Anfang von 1950/51 mit so vielen Schwierigkeiten zu kämpfen hatte. ${ }^{26}$ Der funktionelle Ansatz des Schuman-Plans, der auf die Folgewirkungen des Beginnens an einem ,,begrenzten, aber entscheidenden Punkt" setzte, ${ }^{27}$ war insofern nicht ganz unschuldig an diesen Schwierigkeiten, als er eine baldige Korrektur der britischen Haltung infolge der grundlegenden Veränderung der Gesamtkonstellation europäischer Politik suggerierte und so viele Europäer zögern ließ, auch die nächsten Schritte ohne britische Beteiligung zu gehen. Möglicherweise hätte eine offensivere politische Pädagogik der Öffentlichkeit der Sechs zu einer realistischeren Wahrnehmung der britischen Haltung verhelfen können.

\section{Erkenntnisse für die Zukunft}

Aber auch wenn sich der Weiterbau an der europäischen Konstruktion als schwierig erweisen sollte, erwies sich der Grundstein der EGKS als durchaus tragfähig. Insofern steckt

22 Vgl. etwa die lebhafte Kritik von Edmund Dell: The Schuman Plan and the British abdication of leadership in Europe, Oxford 1995.

23 Vgl. Heinen: Frankreich, 1998, S. 73-74.

24 Anne Dulphy/Christine Manigand: Du Plan Schuman à la CECA: les perspectives de l'opinion française, in: Wilkens: Plan Schuman, 2004, S. 245-267.

25 Wie Wolfram Kaiser nachgewiesen hat: Wolfram Kaiser: Christian Democracy and the Origins of European Union, Cambridge 2007, S. 191-252.

26 Vgl. Wilfried Loth: Der Abschied vom Europarat. Europapolitische Entscheidungen im Kontext des SchumanPlans, in: Schwabe: Anfänge, 1988, S. 183-195.

27 So Monnet in seinem Memorandum vom 03.05.1950, deutsche Übersetzung in: Ziebura: Deutsch-französische Beziehungen, 1970, S. 195. 
in dem Mythos von den Gründervätern ein wahrer Kern: Der Schuman-Plan stellte tatsächlich den Beginn einer neuen Architektur für Europa dar. Aus seiner Beobachtung ergeben sich einige zentrale Erkenntnisse hinsichtlich der Wirkungsweise der Europäischen Union:

Erstens: Die verbreitete Gegenüberstellung von europäischem Idealismus und nationalen Egoismen ist wenig sinnvoll. In der europäischen Konstruktion ging und geht es um die Verfolgung gemeinsamer oder komplementärer Interessen der europäischen Nationen, die auf nationalstaatlicher Ebene allein nicht mehr oder nicht so gut bedient werden können. Die Qualität europäischer Politik ergibt sich daraus, wie weit und wie effektiv europäische Regierungen der Einsicht in die begrenzte Kapazität des nationalstaatlichen Instrumentariums folgen.

Zweitens: Kern der europäischen Konstruktion ist der Friedensschluss zwischen den kontinentalen Großmächten Frankreich und Deutschland, wie ihn Winston Churchill schon in seiner Züricher Rede vom September 1946 gefordert hat. ${ }^{28}$ Eine Führungsfunktion in dieser Konstruktion können daher nur die französische und die deutsche Regierung gemeinsam wahrnehmen. Wenn sie effektiv sein wollen, müssen sie allerdings darauf achten, die europäischen Partner nicht hegemonial zu übervorteilen.

Drittens: Den größten Erfolg verspricht immer noch, wie Monnet es formuliert hat, ,eine konkrete und entschlossene Aktion, die auf einen begrenzten, aber entscheidenden Punkt abzielt, im Hinblick darauf eine fundamentale Veränderung nach sich zieht und Schritt für Schritt die einzelnen Bestandteile selbst des Gesamtproblems ändert. "29 Europa, so ergänzte es Schuman in der Erklärung vom 9. Mai 1950, ,wird durch konkrete Tatsachen entstehen, die zunächst eine Solidarität der Tat schaffen. " ${ }^{30}$ Insofern dürfte es hilfreich sein, wenn sich die europapolitische Debatte nach den Jahren ziemlich abstrakter Beschäftigung mit der Reform der europäischen Institutionen wieder aktuellen Sachthemen zuwendet. Die Vorstellung, mit dem Vertrag von Lissabon sei ein Endpunkt europäischer Integration erreicht, erscheint jedenfalls ziemlich weltfremd.

28 Text u.a. in Lipgens/Loth: Documents, 1988, S. 662-666.

29 Zitiert nach Ziebura: Deutsch-französische Beziehungen, 1970, S. 195.

30 Zitiert nach: Möller/Hildebrand: Die Bundesrepublik Deutschland, 1997, S. 225-227. 